\title{
Erfahrungsaustausch und Koordination
}

\author{
Johann Steurer \\ Prof. Dr. med., Delegierter der Medizinischen Fakultät der Universität Zürich für das Bildungsnetzwerk Medizin
}

Die Schweiz braucht mehr Absolventen eines Humanmedizinstudiums. Dies ist die Kernbotschaft eines Sonderprogramms, welches der Bundesrat 2016 verabschiedet hat. Die Universitäten haben darauf reagiert - sei es mit der Erhöhung von Studienplätzen, zeitgemässen Curriculae oder neuen Standorten. Für die Umsetzung dieser Innovationen braucht es eine konstruktive Zusammenarbeit zwischen den Anbietern der Studiengänge. Deshalb haben sich die Studienverantwortlichen der Standorte Zürich, St. Gallen, Luzern, Lugano und Basel zu einem Netzwerk zusammengeschlossen.

In der Schweiz wurden zu wenig Medizinerinnen und Mediziner ausgebildet. Folglich stieg der Anteil an Ärztinnen und Ärzten mit ausländischem Diplom auf etwa ein Drittel an. Doch die Rekrutierung von Medizinern im Ausland wird schwieriger. 2016 lancierte der Bundesrat deshalb ein Sonderprogramm zur nachhaltigen Erhöhung der Anzahl Medizinstudierender und stellte dafür 100 Millionen Franken zur Verfügung. In der Folge erhöhten einerseits die bestehenden Medizinischen Fakultäten die Anzahl an Ausbildungsplätzen. Andererseits bilden nun weitere Universitäten

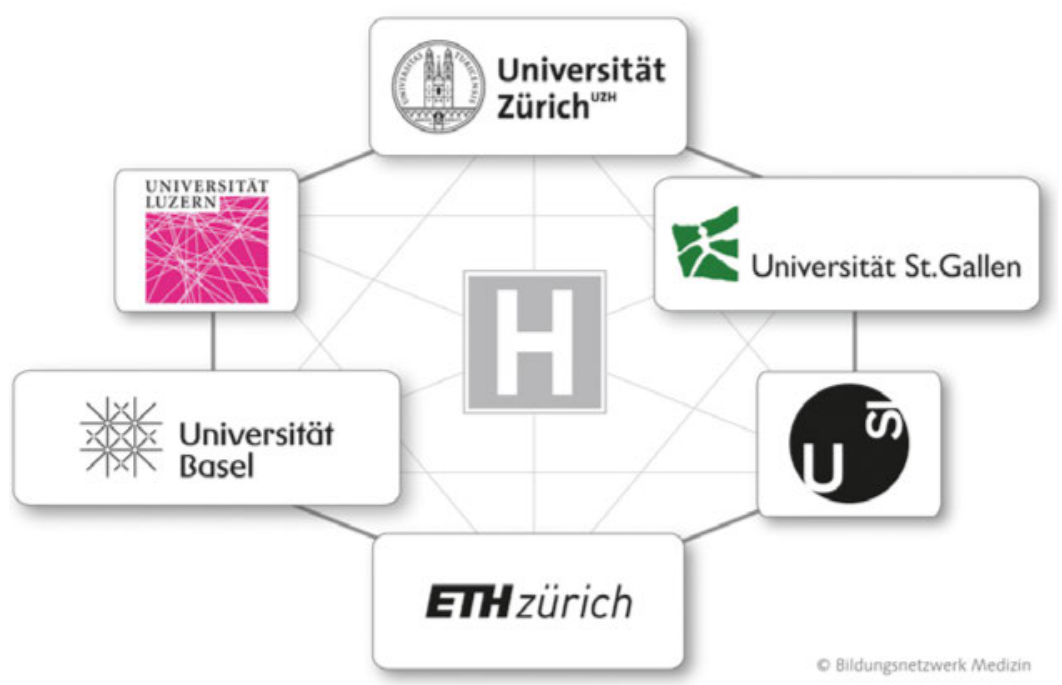

Abbildung 1: Die sechs Partner des Bildungsnetzwerks Medizin.
Mediziner aus. So bietet seit Herbst 2017 die ETH Zürich einen Bachelorstudiengang in Medizin an. Der grösste Teil der Absolventen der ETH wird für das Masterstudium an die Università della Svizzera Italiana (USI) wechseln, und je ein kleinerer Teil an die Universitäten Basel und Zürich. Im Herbst 2017 haben an der Universität Zürich 370 das Bachelorstudium in Medizin begonnen, und je 40 werden das Masterstudium in St. Gallen und Luzern absolvieren. Die Studierenden in Luzern und St.Gallen werden mit einem «Joint Master» abschliessen, der gemeinsam von den Universitäten St. Gallen oder Luzern und Zürich vergeben wird. Luzern und St.Gallen nutzen im Masterstudium zudem die Möglichkeit von Schwerpunktbildungen (Luzern in ambulanter und stationärer Allgemeinmedizin und Gesundheitswissenschaften; St.Gallen in Grundversorgung, Interprofessionalität und Management \& Governance). Das Ziel der «Joint Master»-Programme ist, die Studierenden bereits im Studium mit den lokalen Möglichkeiten vertraut zu machen und sie zu motivieren, nach dem Studium in den entsprechenden Regionen zu arbeiten.

\section{Ein Netzwerk als Informationsdrehscheibe}

Mit den neuen Ausbildungsorten und den notwendigen Wechseln zwischen verschiedenen Universitäten entsteht Koordinationsbedarf. In erster Linie gilt es sicherzustellen, dass die Anschlussfähigkeit der Studierenden an den einzelnen Universitäten garantiert ist. Die Bachelorausbildung an der ETH, um ein Beispiel zu 


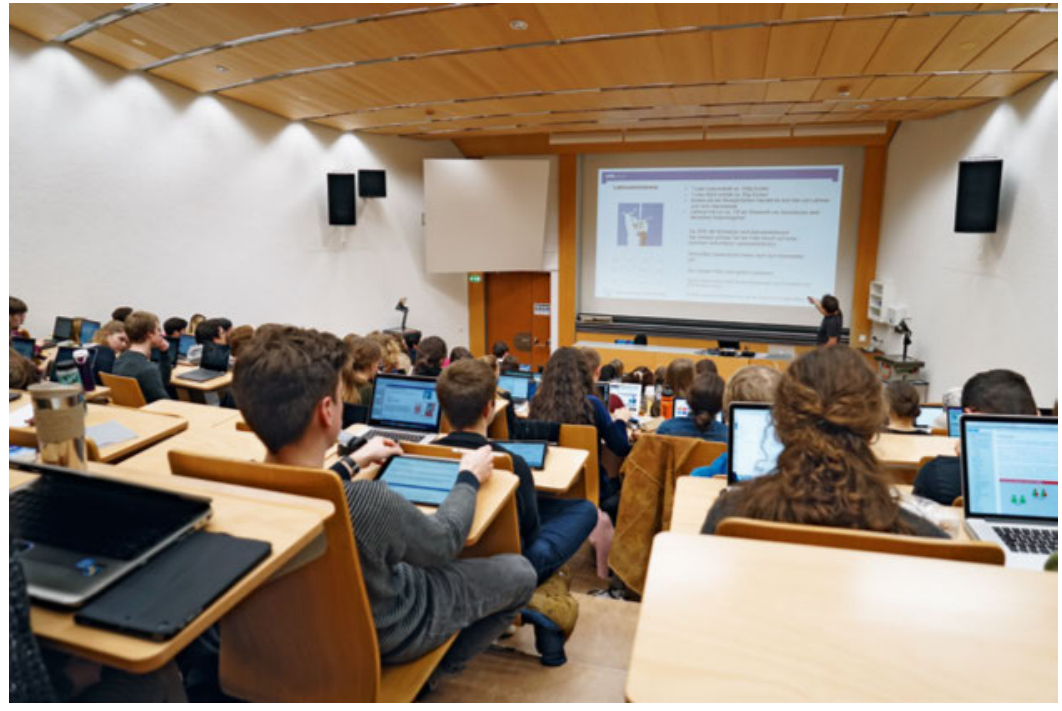

Die ETH Zürich bietet seit Herbst 2017 einen Bachelorstudiengang in Medizin an.

Korrespondenz: Prof. Dr. med. Johann Steurer Pestalozzistrasse 24 CH-8091 Zürich. johann.steurer[at]usz.ch nennen, ist so aufgebaut, dass deren Studierende für ein Masterstudium im Tessin, in Basel und Zürich vorbereitet sind.

Die Partner initiierten deshalb die Bildung eines gemeinsamen Bildungsnetzwerks zwischen den Universitäten Zürich, St.Gallen, Luzern, Lugano und Basel. Jede dieser Institutionen ernannte Delegierte für das Netzwerk. Dieses konstituierte sich im März 2017, und die Mitglieder treffen sich regelmässig (weitere Informationen zum Bildungsnetzwerk Medizin finden sich auf www.medunet.ch).

Das Bildungsnetzwerk dient in erster Linie der gegenseitigen Information und der Sicherstellung der Anschlussfähigkeit vom Bachelor- ins Masterstudium. Ein weiteres Ziel des Netzwerks ist, von den Partnern lernen zu können. Wenn an einer Universität Erfahrungen mit innovativen didaktischen Methoden - wie dem «flipped classroom» oder dem "Lernen durch Scheitern" - gesammelt werden, werden diese Erfahrungen im Netzwerk ausgetauscht und alle können davon profitieren.

\section{Bedürfnisorientierte Weiterentwicklung}

Im Bildungsnetzwerk haben wir aber auch den Anspruch, das Medizinstudium in inhaltlicher, didaktischer und struktureller Form weiterzuentwickeln, um mit den Entwicklungen im Gesundheitswesen Schritt $\mathrm{zu}$ halten. Als Netzwerk werden wir Vorschläge und Empfehlungen entwickeln, die in den Diskussionen über die Ausbildung der Mediziner Gewicht haben werden. Ein erstes gemeinsames Projekt ist, die potentiellen Konsequenzen der Digitalisierung in der Medizin zu antizipieren. Die Digitalisierung wird den Beruf der Mediziner verändern, und die Frage ist, welche Konsequenzen dies auf die Ausbildung haben sollte. Sollte, um ein Beispiel zu nennen, der mehr oder weniger uneingeschränkte und schnelle Zugang zu Informationen einen Einfluss darauf haben oder nicht, was die Studenten auswendig lernen müssen? Eine andere Frage, über die wir nachdenken, ist die Möglichkeit, den Studierenden bereits in der Ausbildung eine Fokussierung auf einen Schwerpunkt (z.B. Psychiatrie, Allgemeinmedizin) zu ermöglichen oder sich künftig bereits im Masterstudium in einer Disziplin, wie es in der Zahnmedizin schon lange üblich ist, zu vertiefen. Wir, die Mitglieder des Bildungsnetzwerks, sind überzeugt, dass wir in diesem Verbund durch gegenseitiges Vertrauen und offenen Informationsaustausch zu einer qualitativ hochstehenden Ausbildung an den beteiligten Standorten beitragen.

\section{Bildnachweise}

Grafik: @ Bildungsnetzwerk Medizin

Foto: Matthias Scholer 